\title{
Hypocapnia after traumatic brain injury: how does it affect the time constant of the cerebral circulation?
}

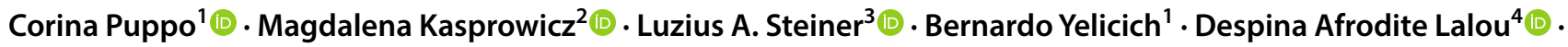 \\ Peter Smielewski ${ }^{4}$ (D) Marek Czosnyka ${ }^{4}$ (i)
}

Received: 12 January 2019 / Accepted: 30 May 2019 / Published online: 7 June 2019

(c) Springer Nature B.V. 2019

\begin{abstract}
The time constant of the cerebral arterial bed ("tau") estimates how fast the blood entering the brain fills the arterial vascular sector. Analogous to an electrical resistor-capacitor circuit, it is expressed as the product of arterial compliance (Ca) and cerebrovascular resistance (CVR). Hypocapnia increases the time constant in healthy volunteers and decreases arterial compliance in head trauma. How the combination of hyocapnia and trauma affects this parameter has yet to be studied. We hypothesized that in TBI patients the intense vasoconstrictive action of hypocapnia would dominate over the decrease in compliance seen after hyperventilation. The predominant vasoconstrictive response would maintain an incoming blood volume in the arterial circulation, thereby lengthening tau. We retrospectively analyzed recordings of intracranial pressure (ICP), arterial blood pressure (ABP), and blood flow velocity (FV) obtained from a cohort of 27 severe TBI patients [(39/30 years (median/IQR), 5 women; admission GCS 6/5 (median/IQR)] studied during a standard clinical $\mathrm{CO}_{2}$ reactivity test. The reactivity test was performed by means of a 50 -min increase in ventilation ( $20 \%$ increase in respiratory minute volume). CVR and Ca were estimated from these recordings, and their product calculated to find the time constant. CVR significantly increased [median CVR pre-hypocapnia/during hypocapnia: $1.05 / 1.35 \mathrm{mmHg} /\left(\mathrm{cm}^{3} / \mathrm{s}\right)$ ]. Ca decreased (median Ca pre-hypocapnia/during hypocapnia: $0.130 / 0.124$ arbitrary units) to statistical significance $(p=0.005)$. The product of these two parameters resulted in a significant prolongation of the time constant (median tau pre-hypocapnia/during hypocapnia: $\left.0.136 \mathrm{~s} / 0.152 \mathrm{~s}, \mathrm{p}^{<} .001\right)$. Overall, the increase in CVR dominated over the decrease in compliance, hence tau was longer. We demonstrate a significant increase in the time constant of the cerebral circulation during hypocapnia after severe TBI, and attribute this to an increase in cerebrovascular resistance which outweighs the decrease in cerebral arterial bed compliance.
\end{abstract}

Corina Puppo

coripuppo@gmail.com

Magdalena Kasprowicz mkasprow@gmail.com

Luzius A. Steiner

Luzius.Steiner@usb.ch

Bernardo Yelicich

beryeli@gmail.com

Despina Afrodite Lalou

alfadt.lalou@gmail.com

Peter Smielewski

ps10011@cam.ac.uk

Marek Czosnyka

mc141@medschl.cam.ac.uk
1 Intensive Care Unit, Hospital de Clinicas, Universidad de la Republica, Av. Italia s/n, 11600 Montevideo, Uruguay

2 Department of Biomedical Engineering, Faculty of Fundamental Problems of Technology, Wroclaw University of Science and Technology, wybrzeże Stanisława Wyspiańskiego 27, 50-370 Wroclaw, Poland

3 Surgical Intensive Care, Prehospital Emergency Medicine and Pain Therapy, University Hospital Basel, University of Basel, Spitalstrasse 21, 4031 Basel, Switzerland

4 Division of Academic Neurosurgery, Department of Clinical Neurosciences, Addenbrooke's Hospital, University of Cambridge, Hills Rd, Cambridge CB2 0QQ, UK 


\section{Introduction}

The time constant (tau) of the cerebral arterial bed estimates the time (in seconds) required for a blood pulse to fill the arterial cerebral bed distal to the point of insonation. Its calculation has been described using mathematical transformations of arterial blood pressure (ABP) and transcranial Doppler (TCD) cerebral blood flow velocity waveforms, and as a product of brain arterial compliance (Ca) and resistance (CVR) [1, 2]. The time constant has been studied in different situations: vasospasm in subarachnoid haemorrhage [3], hydrocephalus [4] carotid stenosis [2], and hypercapnia [5]; it has also been studied in TBI patients [6] and compared in different vascular brain territories in normal volunteers [7].

How this combination of hypocapnia and trauma affects tau has not been addressed so far. Studying 34 healthy volunteers during changes in ventilation, Kasprowicz et al. [1] showed a prolongation of tau during hypocapnia, related to the increase in cerebrovascular resistance elicited by hyperventilation. On the other hand, it has been shown that cerebral arterial bed compliance decreases after hyperventilation in normal subjects [8] and in TBI patients [9]. This effect could influence tau in the opposite direction, shortening it. In normo-ventilated TBI patients, a decrease in arterial cerebral compliance has also been observed [10].

What would happen with tau in TBI after hyperventilation? We hypothesized that the intense vasoconstrictive action of hypocapnia would dominate over the decrease in compliance seen after hyperventilation in TBI patients. This vasoconstrictive response would maintain an incoming blood volume in the arterial circulation over a longer period, thereby increasing tau.

\section{Patients and methods}

\subsection{Patients}

We retrospectively analyzed recordings of intracranial pressure (ICP), arterial blood pressure (ABP), and transcranial Doppler blood flow velocity (FV) from 27 severe TBI patients: 5 women, 22 men, median age 39 years (range: 17-70 years; IQR: 30 years), median GCS at admission: 6, (range 3-12), in whom an increase in ventilation ( $20 \%$ increase in respiratory minute volume) was performed for $50 \mathrm{~min}$ as part of a standard clinical $\mathrm{CO}_{2}$ reactivity test. The study protocol was presented prospectively to the multidisciplinary NCCU user committee to address ethical issues concerning the publication of these data, which were routinely collected as a part of standard clinical management. Since data were fully anonymized, there were no data protection issues involved; it was agreed that formal informed consent was not required for this report. Studies were performed on day $4 \pm 3$ after injury. Subjects were part of a previously- published prospective study about the time course of cerebral blood flow velocity and intracranial pressure after moderate hyperventilation [11]. The characteristics of the patients are shown in Table 1. The same cohort has been studied in a retrospective analysis of cerebral arterial compliance [9], but tau has not been addressed so far.

\subsection{Management and monitoring}

During the study, all patients were intubated and sedated with propofol (2-5 mg/kg h iv) and fentanyl (1-2 $\mu \mathrm{g} / \mathrm{kg} \mathrm{h}$ iv), and paralyzed with iv atracurium. Patients were managed according to a cerebral perfusion pressure (CPP)orientated protocol, maintaining CPP $<70 \mathrm{~mm} \mathrm{Hg}$ and $\mathrm{ICP}>25 \mathrm{~mm} \mathrm{Hg}$ [11]. ABP was measured invasively with an intra-arterial catheter inserted in the radial artery. ICP was monitored using an intraparenchymal probe (Codman MicroSensor ICP Transducer, Codman \& Shurtleff, Raynham, M.A., USA). Flow velocity (FV) of the middle cerebral artery (MCA) was measured using transcranial Doppler ultrasonography (Multi Dop X4, DWL Elektronische Systeme, Sipplingen, Germany). End-tidal $\mathrm{CO}_{2}\left(\mathrm{EtCO}_{2}\right)$ was monitored using mainstream capnography (Marquette solar 8000 M; Medelco, Boynton Beach, F.L., USA). Data were continuously monitored and recorded simultaneously using analog-to-digital conversion and waveform time integration. The "Intensive Care Monitor ("ICM +")" software, Cambridge Enterprise [12] was used to perform data processing and analysis.

\subsection{Intervention}

To perform $\mathrm{CO}_{2}$ reactivity testing, the ventilator minute volume was increased by $15-20 \%$ after acquiring baseline data for $20 \mathrm{~min}$. The lowest allowed $\mathrm{PaCO}_{2}$ was $26 \mathrm{~mm} \mathrm{Hg}$ and the lowest allowed jugular bulb saturation was $55 \%$. If these values were reached, hyperventilation was immediately stopped. A 10-min stabilization period was followed by a 50-min stable hyperventilation period during which it was unchanged. $\mathrm{PaCO}_{2}$ values were determined by arterial blood gas analysis at the beginning and at the end of the stable hyperventilation period to validate $\mathrm{EtCO}_{2}$ values.

The values pre- and during hypocapnia used in this paper were taken from the basal period and the stable hypocapnia period (Fig. 1). 
Table 1 Characteristics of the patients

\begin{tabular}{|c|c|c|c|c|c|c|c|}
\hline $\mathrm{n}$ & Age & Sex & Day & GCS & Marshall & GOS & GOSE \\
\hline 1 & 42 & $\mathrm{~m}$ & 8 & 7 & DII & & \\
\hline 2 & 18 & $\mathrm{f}$ & 2 & 7 & DII & 4 & 6 \\
\hline 3 & 43 & $\mathrm{~m}$ & 4 & 11 & EML & 4 & 5 \\
\hline 4 & 19 & $\mathrm{~m}$ & 2 & 3 & DI & 4 & 5 \\
\hline 5 & 49 & $\mathrm{~m}$ & 2 & 5 & DII & 1 & 1 \\
\hline 6 & 45 & $\mathrm{~m}$ & 2 & 6 & EML & 3 & 3 \\
\hline 7 & 35 & $\mathrm{f}$ & 6 & 3 & DII & 3 & 3 \\
\hline 8 & 53 & $\mathrm{f}$ & 2 & 9 & DIII & 3 & 3 \\
\hline 9 & 38 & $\mathrm{~m}$ & 4 & 4 & DIII & & \\
\hline 10 & 26 & $\mathrm{~m}$ & 3 & 5 & EML & 4 & 5 \\
\hline 11 & 39 & $\mathrm{~m}$ & 2 & 4 & EML & 3 & 3 \\
\hline 12 & 29 & $\mathrm{~m}$ & 3 & 4 & DII & 4 & 6 \\
\hline 13 & 47 & $\mathrm{~m}$ & 10 & 3 & DII & 3 & 3 \\
\hline 14 & 22 & $\mathrm{~m}$ & 3 & 3 & DII & 5 & 7 \\
\hline 15 & 53 & $\mathrm{~m}$ & 1 & 6 & DI & 4 & 5 \\
\hline 16 & 55 & $\mathrm{~m}$ & 12 & 10 & EML & 5 & 7 \\
\hline 17 & 17 & $\mathrm{f}$ & 5 & 8 & NEML & 4 & 6 \\
\hline 18 & 22 & $\mathrm{~m}$ & 3 & 4 & DI & & \\
\hline 19 & 20 & $\mathrm{~m}$ & 2 & 8 & DII & 5 & 8 \\
\hline 20 & 65 & $\mathrm{~m}$ & 3 & 3 & DII & 1 & 1 \\
\hline 21 & 37 & $\mathrm{~m}$ & 2 & 3 & EML & 1 & 1 \\
\hline 22 & 25 & $\mathrm{~m}$ & 2 & 8 & DII & 5 & 7 \\
\hline 23 & 70 & $\mathrm{~m}$ & 1 & 12 & NEML & 4 & 6 \\
\hline 24 & 23 & $\mathrm{~m}$ & 4 & 8 & EML & 3 & 3 \\
\hline 25 & 64 & $\mathrm{~m}$ & 2 & 8 & EML & 3 & 3 \\
\hline 26 & 47 & $\mathrm{~m}$ & 4 & 3 & DII & 1 & 1 \\
\hline 27 & 50 & $\mathrm{f}$ & 6 & 6 & EML & & \\
\hline Total & 27 & 27 & 27 & 27 & 27 & 23 & 23 \\
\hline Median & 39 & & 2 & 6 & & 4 & 5 \\
\hline IQR & 30 & & 2 & 5 & & 1 & 3 \\
\hline
\end{tabular}

Patients characteristics and outcomes

$n$ patient number; GCS glasgow coma scale; Marshall traumatic data bank classification score; DI diffuse injury I; DII diffuse Injury II; DIII diffuse Injury III; $E M L$ evacuated mass lesion; $N E M L$ non-evacuated mass lesion; GOS glasgow outcome score; GOSE glasgow outcome score extended; IQR interquartile range

\subsection{Data analysis}

The cerebrovascular time constant of the arterial bed was estimated before and during hypocapnia as the product of arterial compliance and cerebrovascular resistance:

$\operatorname{tau}=\mathrm{Ca} * \mathrm{CVR}[\mathrm{s}]$

Cerebral compliance was estimated as the ratio of the pulse amplitude of the cerebral arterial blood volume $(\mathrm{CaBV})$ and the pulse amplitude of ABP.

The CaBV model is based on the assumption that the change in intravascular volume over one cardiac cycle corresponds to the difference between arterial inflow and venous outflow $[13,14]$. Since the venous outflow has a low pulsatility compared to arterial inflow [15], it was assumed to be constant and was calculated using the timeaveraged mean CBFV and the unknown cross-sectional area of the insonated artery. CVR was estimated as the ratio of mean $\mathrm{CPP}(\mathrm{CPP}=\mathrm{ABP}-\mathrm{ICP})$ and mean $\mathrm{CBF}$ was calculated as: $(\mathrm{CBF}=\mathrm{CBFV} * \mathrm{Sa})$, with $\mathrm{Sa}$ noted as unknown but stable cross area of the insonated vessel.

Details of the formula derivation are described elsewhere $[1,2,16]$ and summarized in the appendix.

The distribution of numeric variables $(\mathrm{n}<30)$ was considered non-parametric. Therefore, the Wilcoxon signedrank test was used for comparisons. Statistical significance was assumed at $p<0.05$. SPSS 19 software was used for all calculations. 

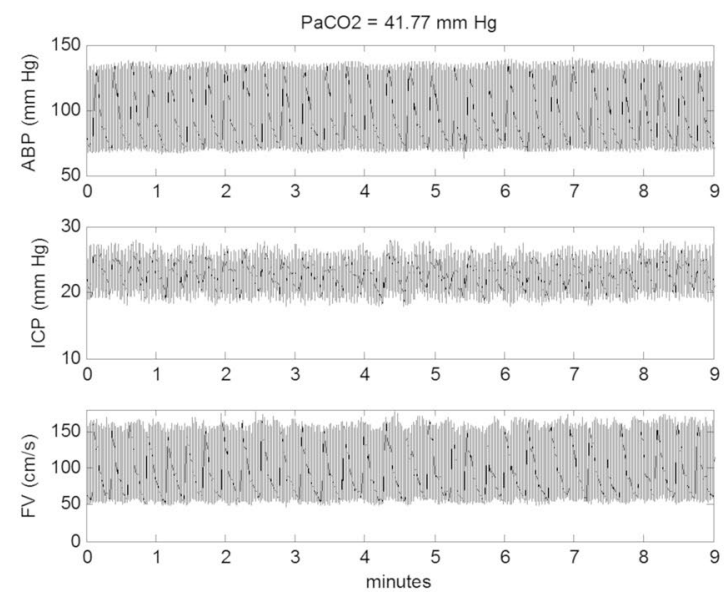

Fig. 1 Raw data acquired at $50 \mathrm{~Hz}$, from one of the patients, showing pre-hypocapnia (left) and hypocapnia (right) stable periods which were analyzed. Note the decrease in ICP and FV during hypocapnia.

\section{Results}

Clinical data, CT findings, and outcomes are summarized in Table 1.

\subsection{Change in $\mathrm{PaCO}_{2}$}

Median (IQR) basal $\mathrm{PaCO}_{2}$ was 38.2 (4.6) mm Hg. During hyperventilation, it was 32.9 (6.9) $\mathrm{mmHg}$. There was a significant difference between both variables (Wilcoxon signed-rank test, $p<0.001$.)

\subsection{Change in cerebrovascular resistance}

CVR increased in 24 out of 27 cases. Basal values median (IQR) were 1.1(1.1) arbitrary units (AU) hypocapnia values were 1.4(0.9) (AU) (Fig. 2, left). The increase was
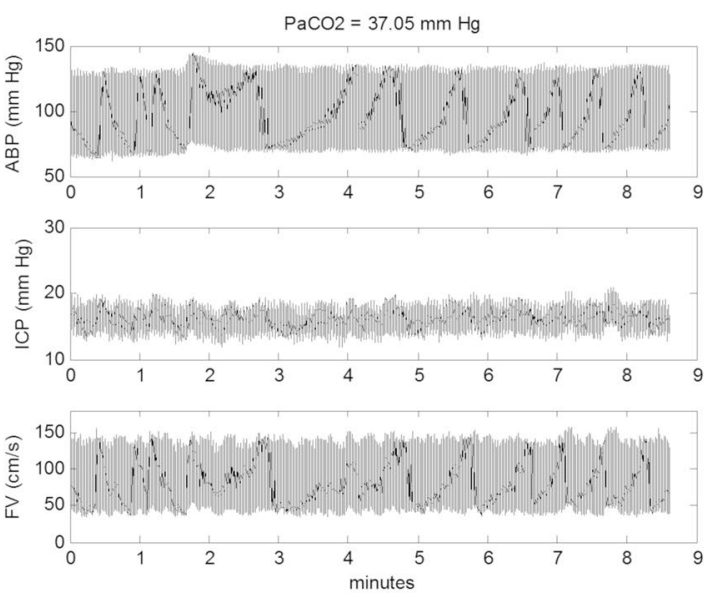

FV: Cerebral blood flow velocity; ICP: intracranial pressure; $A B P$ arterial blood pressure

significant (Wilcoxon signed-rank test, $\mathrm{p}<0.001$ ). The percentage increase in CVR was (median (IQR): $22.6(26.3) \%$.

\subsection{Change in compliance}

Arterial compliance showed a significant decrease (Wilcoxon signed-rank test, $p=0.005$ ) (Fig. 2, center). The percentage decrease [median (IQR)] was $7.0(20) \%$.

\subsection{Change in tau}

Tau got significantly longer during hypocapnia (Wilcoxon signed-rank test, $p<0.001)$. Median (IQR): 0.136(0.08) to $0.152(0.08)$ [s] (Fig. 2, right). The overall increase in tau as expressed in percentages was: [median (IQR)] 11.4(12.7) \%. This increase in CVR outweighs the decrease in CA, explaining the prolongation of tau.
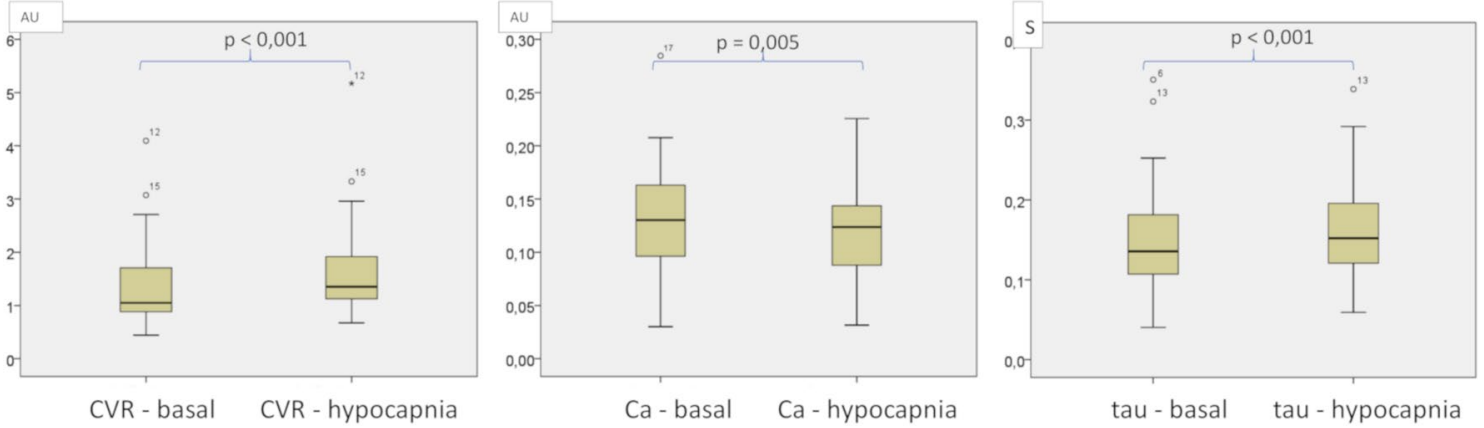

Fig. 2 Change in the different variables during hyperventilation in each patient. Boxplots show the median, minimum, maximum, and interquartile range of $\mathrm{CVR}, \mathrm{Ca}$ and tau, basal and during hyperventilation 


\subsection{Correlations between the different variables changes and $\mathrm{PaCO}_{2}$ change}

The percentage change in CVR (dCVR) and in tau (dtau) before and during hypocapnia plotted against the percentage change in $\mathrm{PaCO}_{2}\left(\mathrm{dPaCO}_{2}\right)$, showed a significant correlation (Fig. 3, upper and lower panels). The correlation between
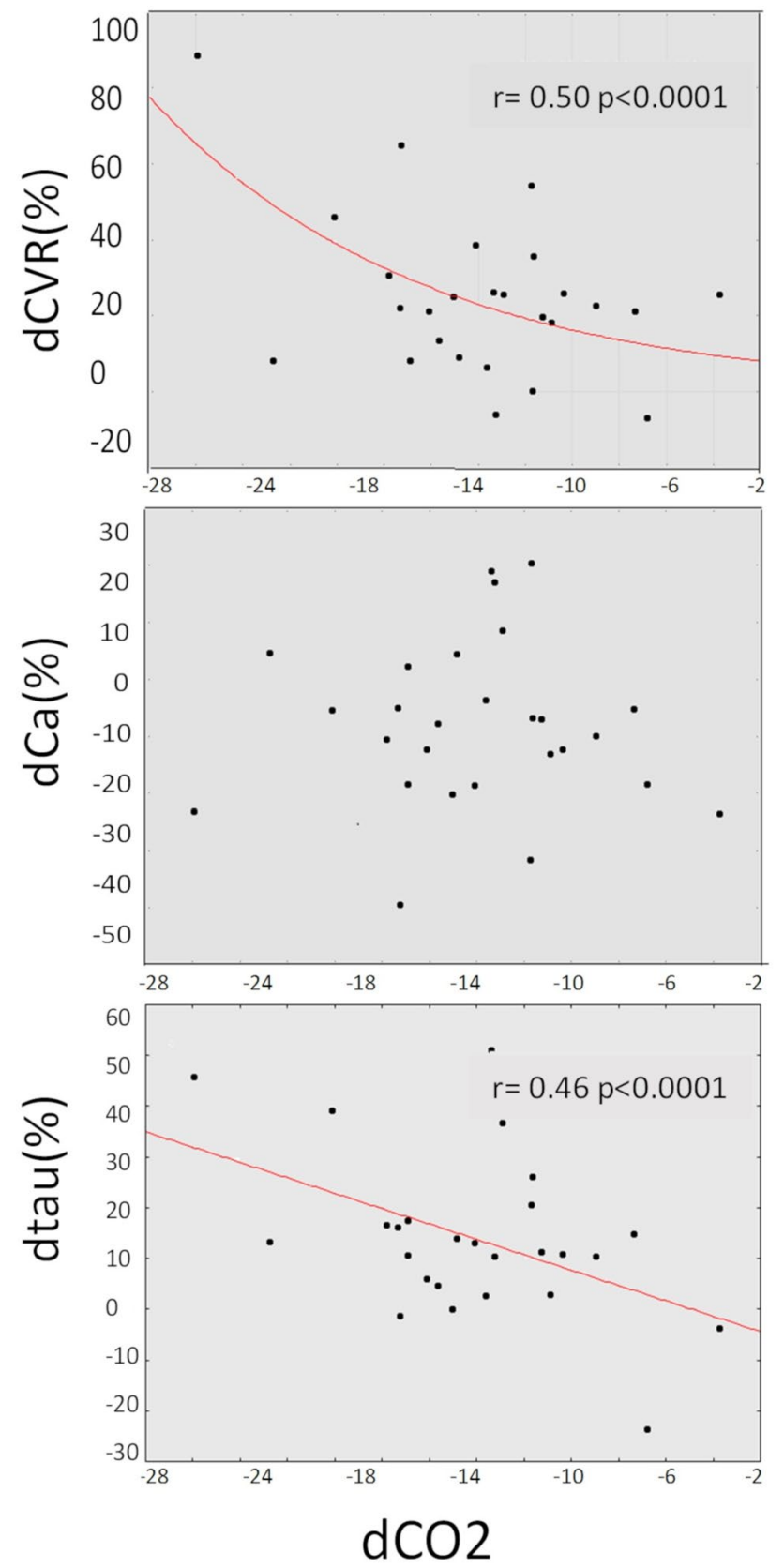

Fig. 3 Change in percentage in CVR (dCVR), Ca (dCa) and tau (dTAU), plotted versus change in paCO2 (dCO2). As can be seen, $\mathrm{dCVR}$ and dTAU are inversely correlated with $\mathrm{dCO} 2$, while $\mathrm{dCa}$ does not show a significant correlation the percentage change in $\mathrm{Ca}(\mathrm{dCa})$ and $\mathrm{dPaCO}_{2}$ was not significant (Fig. 3, center panel).

When the association between $\mathrm{dCVR}$ and $\mathrm{dCa}$ was analyzed, an inverse relationship was found (Fig. 4).

\section{Discussion}

In this work, we detailed the behavior of some of the highfrequency parameters of the cerebral circulation in severe TBI patients during hyperventilation $(\mathrm{Ca}, \mathrm{CaBV}$, tau). TCD FV, ICP, and intraarterial pressure monitoring allows researchers to access pulsatile data from the brain. In this same cohort of patients, other high-frequency parameters, cerebral arterial compliance [9], and cerebral critical closing pressure [17] have been estimated in previous works.

The time constant (tau) of the cerebral arterial bed, determined as a product of vascular resistance and compliance, theoretically reflects how fast an arterial blood load (which reaches the brain as the volume of an arterial pulse) can pass through the cerebral arterial vasculature during a cardiac cycle (from the point of insonation to the arterio-capillary boundary). In the current paper, it has been studied invasively, using FV, ABP, and ICP signals. Tau (expressed in seconds) has the advantage to be independent of the cross-sectional diameter of the insonated artery, a value which cannot be measured with TCD. The values found in normal subjects are: [median, (IQR)): 0.22, (0.17,

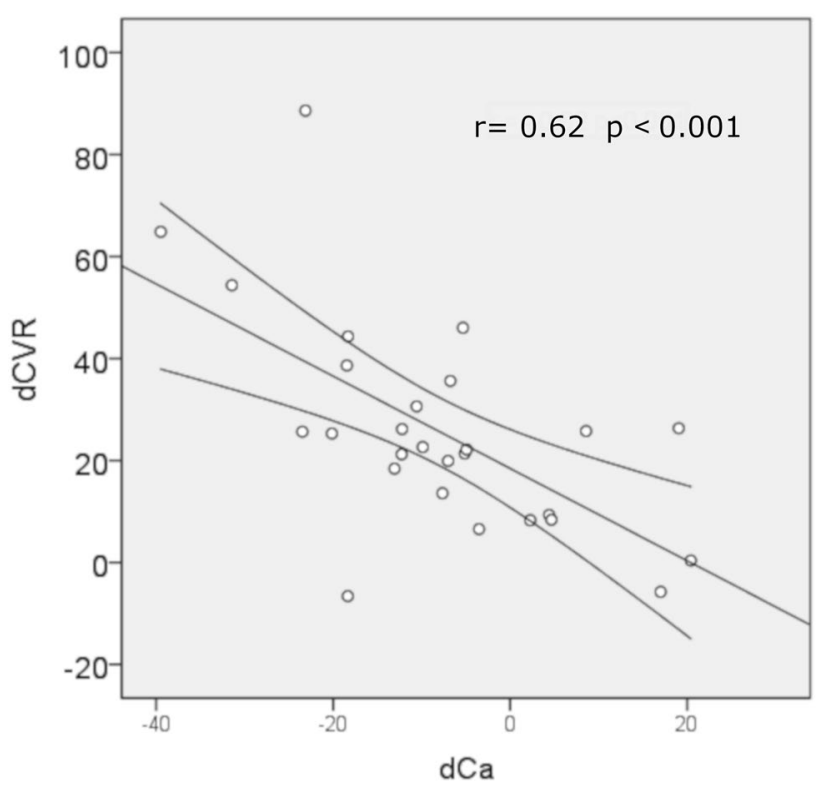

Fig. 4 Inverse proportionality between the change in CVR (dCVR) and the change in $\mathrm{Ca}(\mathrm{dCa})$. These inverse changes could affect in opposite directions the change in tau. However, the change in CVR predominated, resulting in a change in tau in the same direction as CVR (increase) 
0.26) seconds for young normal subjects] [1], but it has also been expressed as a percentage between $20-30 \%$ of the cardiac cycle in normal volunteers $[1,4]$. It has been demonstrated that the time constant gets longer during hypocapnia in healthy volunteers through an increase in CVR [1], and shortens in patients with traumatic brain injury through a decrease in $\mathrm{Ca}[6]$.

It has also been studied in subarachnoid hemorrhage, showing that vasospasm, which decreases compliance and generates a reactive microcirculatory vasodilation distal to the diminished vessel radius, shortens the time constant [3]. These significantly shorter values appear before other TCD indices (mean flow velocity and the Lindegaard Ratio) could diagnose it.

Czosnyka et al. [5] analyzed the change in tau in $46 \mathrm{New}$ Zealand rabbits after different modifications in systemic or cerebral pressures or in respiratory variables. The registered signals were: arterial pressure, $\mathrm{EtCO}_{2}$, intracranial pressure, and TCD cerebral blood flow velocity. The animals were divided in five groups depending on the performed change. In one of the groups, $\mathrm{EtCO}_{2}$ was changed in 9 animals between $35-55 \mathrm{~mm} \mathrm{Hg}$ by adjusting the tidal volume of the ventilator. The changes in ventilation also generated changes in arterial blood pressure in some cases, which made the interpretation of the hypocapnia action more difficult. However, in 6 animals the changes in arterial pressure were limited, demonstrating an inverse response in CVR in relation to $\mathrm{EtCO}_{2}$ change. When studying the animals together with empirical regression, the increase in $\mathrm{EtCO}_{2}$ elicited a decrease in CVR. CVR decreased on average to $65 \%(p<0.05)$ of the value observed at an $\mathrm{EtCO}_{2}$ of $35 \mathrm{~mm}$ $\mathrm{Hg}$ (lower normocapnia). On the other hand, Ca increased, but at a lesser rate. As a result, tau got shorter. These results are comparable with the changes that we observed in our cohort.

In this study, we found: (a) tau (both basal and hypocapnic values) was shorter in TBI patients than in normal volunteers. However, the calculation performed in normal volunteers does not include ICP in its formula, therefore, a statistical comparison cannot be performed; (b) When hypocapnia was induced, significant changes were elicited. Tau got significantly longer. This was related to (c) a greater change in CVR, which increased, compared to (d) the change in $\mathrm{Ca}$, which decreased. This decrease in Ca had already been found in this same group of patients by Carrera et al. [9]. This research hypothesized that the reduction in Ca during hypocapnia is related to an increase in arterial microvascular tone; this reduces the ability of the arterial bed to compensate for added cerebral arterial blood volume in each arterial pulse. Simultaneously, the decrease in CBV caused by vascular tree vasoconstriction moves the point where the brain is working in the pressure-volume curve to a less steep position.
Our results showed that in severe TBI patients, hypocapnia adjusts $\mathrm{CBF}$ through direct changes in $\mathrm{CVR}$, with $\mathrm{Ca}$ being the rate of change of volume with pressure (indirectly affected). As a result, the change in the time constant is essentially primarily driven by the change in CVR.

Tau is potentially a very useful and interesting biomarker. Apart from the asymmetric values found during vasospasm in $\mathrm{SAH}$, in non-asymmetric pathology a progressive increase of its value could make the physician aware that at least one of the two factors implied in its calculation can be changing either resistance or compliance.

There were some limitations to the study. First, some assumptions have been made in pulsatile volume modeling, specially that the venous outflow remains constant throughout the cardiac cycle. Actually, cerebral blood outflow is pulsatile; however, the magnitude of these venous pulsations is much lower than arterial pulsations $[15,18,19]$. This circumstance allows the use of a constant flow instead of an unknown low pulsatility one.

Another assumption is about vascular cross-sectional area invariability. The area of the insonated large vessel, the MCA, is not constant [20-22]. Large vessel diameters examined directly during surgery [20] or using 3T MRI [21], show a certain variability under different stimuli (like changes in $\mathrm{CO}_{2}$ ), though much smaller than those of arterioles or precapillary sphincters). This variability does cause some changes in $\mathrm{Ca}$ and CVR, but we have to underline that the calculation of tau makes it independent of this value.

Further, the number of patients investigated in this retrospective study was small. However, this population was sizeable enough to demonstrate that the increase in CVR elicited by hypocapnia was greater in percentage (and therefore had more impact on the increase of tau) than the decrease in cerebral arterial bed compliance elicited by trauma. A larger prospective study would be needed to further establish its potential usefulness in clinical practice. Finally, the model used in this trial has been validated with other monitoring or imaging techniques, emphasizing its validation with perfusion $\mathrm{CT}$ in trauma patients [10].

\section{Conclusions}

In TBI patients who were hyperventilated in a routine test of $\mathrm{CO}_{2}$ reactivity, we could find a significant prolongation of the time constant of the cerebral circulation due to an increase in CVR, which overrode the simultaneously-induced decrease in compliance of the cerebral arterial bed. 


\section{Appendix}

\subsection{Calculation of compliance}

To calculate the compliance of the cerebral arterial bed, one must know: (1) how the cerebral arterial blood volume $(\mathrm{CaBV})$ changes with each pulsatile inflow of arterial blood, and (2) the arterial blood pressure which generates this volume change.

\subsection{Calculation of cerebral arterial blood volume}

The estimation of CaBV is based on a modification [1-3, 16] of Avezaat and van Eijndhoven's concept [13, 14] (Fig. 5). The change in blood volume ( $\triangle \mathrm{CaBV}$ ) during an incoming arterial pulse can be calculated as the integral of the difference between pulsatile arterial inflow (CaBF) and low-pulsatility venous outflow (CvBF).

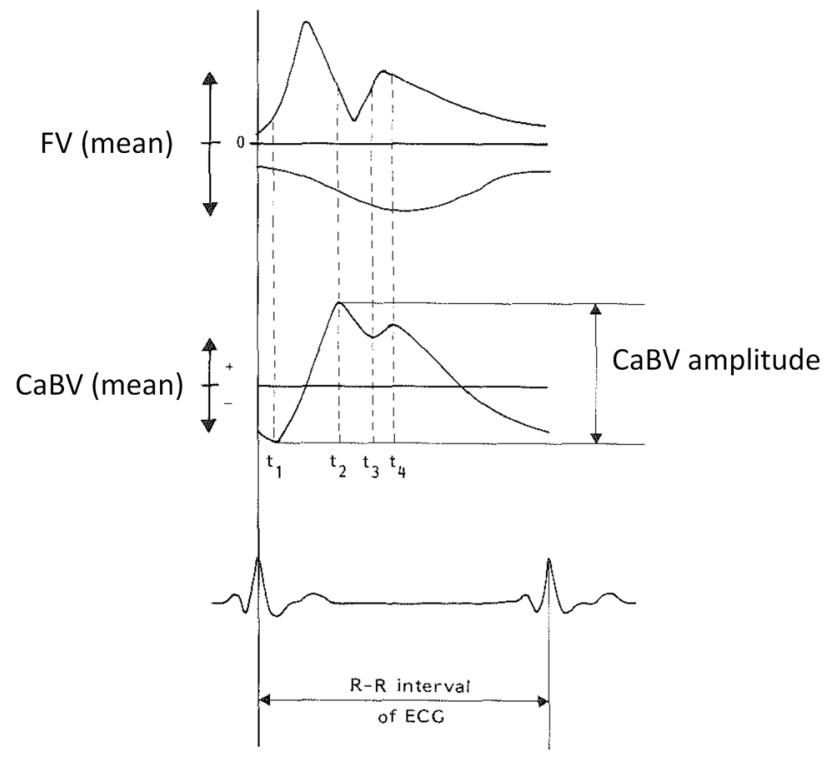

Fig. 5 Comparison of FV and CaBV [13]. (Reproduced with permission from Erasmus University). The upper curve shows the cerebral blood velocity profile as seen with TCD sonography. The arterial velocity profile is above zero and the venous is below zero. Note the lower pulsatility of the venous velocity profile, which therefore can be approximated by a constant flow. The lower curve shows the $\mathrm{CaBV}$ as calculated with the above described model. Note that the volume keeps increasing when the velocity has already started to fall. The ECG is shown below the two curves to facilitate the understanding of each pulse profile. The timing between the pulsatile inflow and outflow curves with the resultant change in CBV during a cardiac cycle is schematically demonstrated in the figure. The arterial inflow equals the venous outflow at times $\mathrm{t} 2, \mathrm{t} 3$ and $\mathrm{t} 4$. The blood volume increases when inflow exceeds outflow, (intervals $\mathrm{t} 1-\mathrm{t} 2$ and $\mathrm{t} 3-\mathrm{t} 4$ ) and decreases in the reverse situation. $\mathrm{t} 1$ and $\mathrm{t} 2$ respectively correspond to the minimum and maximum volume
The assumption is made that the low-pulsatility venous outflow may be approximated by a constant flow equal to the averaged arterial inflow (CBFm). The instantaneous averaged arterial inflow in the insonated point is equal to the mean cerebral blood flow velocity (FVm) multiplied by the cross-sectional area of the insonated vessel (Sa). Accounting for finite sampling frequency, we can write a discrete time difference equation in terms of flow velocity (FV).

$\mathrm{CaBV}(\mathrm{n})=\sum_{i=1}^{n}\left(\mathrm{FV} i * \frac{1}{\mathrm{AF}} * S a-\mathrm{FV} m * \frac{1}{\mathrm{AF}} * S a\right)$

$\mathrm{Sa}=$ cross sectional area of the insonated vessel (MCA) (unknown), 1/AF = Acquisition frequency (AF) period., $\mathrm{n}=$ number of samples during the cardiac cycle, Eq. 1 can be simplified as:

$\Delta \mathrm{CaBV}(n)=\frac{\mathrm{Sa}}{\mathrm{AF}} \sum_{l=1}^{n}(\mathrm{FV} i-\mathrm{FVm})$

The compliance of the cerebral arterial bed $(\mathrm{Ca})\left[\left(\mathrm{cm}^{3} /\right.\right.$ $\mathrm{mmHg})]$ is calculated as the quotient of $\mathrm{CaBV}$ amplitude by ABP amplitude.

$\mathrm{Ca}=\mathrm{CaBV} 1 / \mathrm{ABP} 1$

$\mathrm{CaBV}$ amplitude (CaBV1) is the maximum volume during the heartbeat minus the minimum volume at the end of the diastolic phase of the velocity envelope. It is calculated as the first harmonic of the CaBV curve using a Fast Fourier transformation.

$\mathrm{ABP}$ amplitude (ABP1) is the maximum pressure during the heartbeat minus the minimum pressure at the end of the diastolic phase of the arterial pressure pulse. It is calculated as the first harmonic of the ABP curve using a Fast Fourier transformation.

Cerebrovascular Resistance (CVR) $\left[\mathrm{mmHg} /\left(\mathrm{cm}^{3 *} \mathrm{~s}\right)\right]$ is estimated as the ratio between mean cerebral perfusion pressure $(\mathrm{mCPP}=\mathrm{mABP}-\mathrm{mICP})$ and cerebral blood flow $(\mathrm{CBF})$. $\mathrm{CBF}$ is calculated as the mean cerebral blood flow velocity multiplied by the unknown but stable cross-sectional area of the insonated artery $(\mathrm{Sa})$.

$\mathrm{CBF}=\mathrm{FVm} * \mathrm{Sa}$

$\mathrm{CVR}=\frac{\mathrm{CPPm}}{\mathrm{FVm} * \mathrm{Sa}}$

Note that in the CVR formula, the unknown but stable cross-sectional area of insonated artery is in the divisor.

\subsection{Calculation of the cerebral arterial time constant (tau)}

$\operatorname{tau}=\mathrm{CVR} * \mathrm{Ca}$ 
The product of $\mathrm{Ca}$ (Eq. 3) and CVR (Eq. 5) removes the unknown contribution from the cross-sectional area (multiplying in $\mathrm{Ca}$ and dividing in $\mathrm{CVR}$ ) entirely, thus allowing tau to be measured in seconds.

\section{Compliance with ethical standards}

Conflict of interest The authors declare that the research was conducted in the absence of any commercial or financial relationships that could be construed as a potential conflict of interest. MC and PS have financial interest in a part of licensing fee for ICM+software (Cambridge Enterprise Ltd. UK).

\section{References}

1. Kasprowicz M, Diedler J, Reinhard M, Carrera E, Steiner LA, Smielewski P, Budohoski KP, Haubrich C, Pickard JD, Czosnyka $\mathrm{M}$. Time constant of the cerebral arterial bed in normal subjects. Ultrasound Med Biol. 2012;38:1129-37. https://doi.org/10.1016/j. ultrasmedbio.2012.02.014

2. Kasprowicz M, Diedler J, Reinhard M, Carrera E, Smielewski P, Budohoski KP, Sorrentino E, Haubrich C, Kirkpatrick PJ, Pickard JD, Czosnyka M. Time constant of the cerebral arterial bed. Acta Neurochir Suppl. 2012;114:17-21. https://doi. org/10.1007/978-3-7091-0956-4_4.

3. Kasprowicz M, Czosnyka M, Soehle M, et al. Vasospasm shortens cerebral arterial time constant. Neurocrit Care. 2012;16:213-8.

4. Capel C, Kasprowicz M, Czosnyka M, et al. Cerebrovascular time constant in patients suffering from hydrocephalus. Neurol Res. 2014;36:255-61.

5. Czosnyka M, Richards HK, Reinhard M, et al. Cerebrovascular time constant: dependence on cerebral perfusion pressure and endtidal carbon dioxide concentration. Neurol Res. 2012;34:17-24.

6. Trofimov A, Kalentiev G, Gribkov A, Voennov O, Grigoryeva V. Cerebrovascular time constant in patients with head injury. Acta Neurochir Suppl. 2016;121:295-7.

7. Kasprowicz M, Czosnyka M, Poplawska K, Reinhard M. Cerebral arterial time constant recorded from the MCA and PICA in normal subjects. Acta Neurochir Suppl. 2016;122:211-4.

8. Carrera E, Kim DJ, Castellani G, et al. Effect of hyper- and hypocapnia on cerebral arterial compliance in normal subjects. J Neuroimaging. 2011;21:121-5.

9. Carrera E, Steiner LA, Castellani G, et al. Changes in cerebral compartmental compliances during mild hypocapnia in patients with traumatic brain injury. J Neurotrauma. 2011;28:889-96.

10. Dobrzeniecki M, Trofimov A, Bragin DE. Cerebral Arterial Compliance in Traumatic Brain Injury. Acta Neurochir Suppl. 2018;126:21-4.
11. Steiner LA, Balestreri M, Johnston AJ, et al. Sustained moderate reductions in arterial $\mathrm{CO}_{2}$ after brain trauma time-course of cerebral blood flow velocity and intracranial pressure. Intensive Care Med. 2004;30:2180-7.

12. Smielewski P, Czosnyka Z, Kasprowicz M, Pickard JD, Czosnyka $\mathrm{M}$. ICM + : a versatile software for assessment of CSF dynamics. Acta Neurochir Suppl. 2012;114:75-9.

13. Avezaat CJJ, Eijndhoven JHM (1984). Cerebrospinal fluid pulse pressure and craniospinal dynamics: A theoretical, clinical and experimental study. the Netherlands: The Hague: Jongbloed en Zoon.

14. van Eijndhoven JH, Avezaat CJ. Cerebrospinal fluid pulse pressure and the pulsatile variation in cerebral blood volume: an experimental study in dogs. Neurosurgery. 1986;19:507-22.

15. Aaslid R, Newell DW, Stooss R, Sorteberg W, Lindegaard KF. Assessment of cerebral autoregulation dynamics from simultaneous arterial and venous transcranial doppler recordings in humans. Stroke. 1991;22:1148-54.

16. Varsos GV, Kasprowicz M, Smielewski P, Czosnyka M. Modelbased indices describing cerebrovascular dynamics. Neurocrit Care. 2014;20:142-57.

17. Smielewski P, Steiner L, Puppo C, Budohoski K, Varsos GV, Czosnyka M. Effect of mild hypocapnia on critical closing pressure and other mechanoelastic parameters of the cerebrospinal system. Acta Neurochir Suppl. 2018;126:139-42.

18. Alperin N, Sivaramakrishnan A, Lichtor T. Magnetic resonance imaging-based measurements of cerebrospinal fluid and blood flow as indicators of intracranial compliance in patients with Chiari malformation. J Neurosurg. 2005;103:46-52.

19. Stolz E, Kaps M, Kern A, Babacan SS, Dorndorf W. Transcranial color-coded duplex sonography of intracranial veins and sinuses in adults. Reference data from 130 volunteers. Stroke. 1999;30:1070-5.

20. Giller CA, Bowman G, Dyer H, Mootz L, Krippner W. Cerebral arterial diameters during changes in blood pressure and carbon dioxide during craniotomy. Neurosurgery. 1993;32:737-41.

21. Hoiland RL, Ainslie PN. CrossTalk proposal: the middle cerebral artery diameter does change during alterations in arterial blood gases and blood pressure. J Physiol. 2016;1(594):4073-5.

22. Coverdale NS, Lalande S, Perrotta A, Shoemaker JK. Heterogeneous patterns of vasoreactivity in the middle cerebral and internal carotid arteries. Am J Physiol Heart Circ Physiol. 2015;308:H1030-8.

Publisher's Note Springer Nature remains neutral with regard to jurisdictional claims in published maps and institutional affiliations. 